\section{Hydro-Electric Development in India}

IN NATURE of January 6, p. 23, under this heading, it was stated that an irrigation scheme involving the generation of large quantities of electric power between the Punjab and the State of Bilaspur "is under construction". We are informed by the secretary of the Central Board of Irrigation that "the project referred to, namely the Bhakra Dam Project in the Punjab, is not 'under construction' but simply 'under consideration' at present".

\section{Exhibition of Chinese Art at Leeds}

AN exhibition of Chinese art was opened in the Temple Newsham Mansion, Leeds, on March 23 by Dr. Thomas Bodkin, Barber professor of Fine Arts and director of the Barber Institute in the University of Birmingham. The exhibition consists of more than 200 specimens of pottery, paintings and bronzes, exhibited by the courtesy of Messrs. John Sparks of London, and on the initiative of the curator of Temple Newsham, Mr. Philip Hendy. Although the exhibition is, so far as possible, comprehensive in a chronological sense, the periods which will appeal most to students of the earlier phases of Chinese culture, covering the Shang-Yin (? 1766-1122 B.C.) and Chou (? 1122-249 B.c.) Dynasties and the Warring States, are represented by some notable exhibits forming a group of seven very rare bronzes. Three belong to the Shan-Yin Dynasty-two beakershaped vessels with a finely engraved design, and a sacrificial cup. There are also three vessels of the Chou Dynasty; and a dagger with a handle in the form of a mule's head is of the period of the Warring States (481-221 B.c.). Special interest is attached to these bronzes, all coming from burials. Among the most important of the pottery exhibits is the figure of a Lohan or Buddhist priest, seated with arms folded in the sleeves of his cloak, which stands about four feet high.

\section{A Big Sunspot}

A BIG sunspot has erossed the solar disk during the past week; appearing at the east limb on March 20 , reaching the central meridian on March $26 \cdot 3$ and due to pass around the west limb of the disk on April 1. The area of the spot on March 23 was 1,000 millionths of the sun's hemisphere-an area well above the lower limit of size for naked-eye visibility. On March 23, a bright chromospheric eruption of considerable intensity was observed directly over the spot. A great magnetic storm began on March 23 and was in progress during the night of March 24-25, when the aurora was also seen in spite of bright moonlight. Widespread interference with both radio and cable communications was reported.

\section{The Night Sky in April}

During the month, night shortens by nearly two hours in the latitude of London. The moon is new on April 7 at $20 \cdot 3 \mathrm{~h}$. and full on April 22 at $4 \cdot 6 \mathrm{~h}$.
On the latter date, a penumbral eclipse of the moon occurs, having been preceded on April 7 by an annular eclipse of the sun invisible in Great Britain, but to be seen in the southern parts of the United States of America. The penumbral lunar eclipse on April 22 begins at $2 \mathrm{~h} .27 \mathrm{~m}$., reaches a maximum phase $(0 \cdot 89)$ at $4 \mathrm{~h} .26 \mathrm{~m}$., and ends at $6 \mathrm{~h} .25 \mathrm{~m}$. In the nature of things, a penumbral lunar eclipse is not a spectacular phenomenon, but a difference in brightness between the east and west parts of the moon's disk should be discernible in a naked-eye scrutiny. In the evening skies after sunset, the brilliant planet Venus dominates the other planets still above the horizon-Mars, which is visible for about three hours after sunset, and Saturn, drawing nearer to the sun's position until it is in conjunction on April 24. Jupiter is now too close to the sun for observation, being in conjunction on April 11. It may be noted that also on April 11 at $0 \mathrm{~h}$. Venus is in conjunction with Mars; while at $19 \mathrm{~h}$. the moon, then nearly four days old, will be in conjunction with both these planets. The Lyrid meteors are due during April 19-22, the radiant point being near 104 Herculis. [All times are given in Universal Time; add 1 hour to convert to Summer Time.]

\section{Announcements}

Sir Harold Carpenter, F.R.S., professor of metallurgy in the Royal School of Mines, London, has been awarded the Honda prize of the Japan Metallurgy Society. $\mathrm{He}$ is the first foreigner to receive the award, which consists of a gold cup and $£ 300$.

Prof. A. V. HILc, M.P., F.R.S., has been appointed as assistant air attaché in Washington for special scientific liaison duties. The appointment is temporary, and has been accepted without remuneration.

The Messel Medal of the Society of Chemical Industry has been awarded to Lord Samuel. This medal, one of the two principal awards in the hands of the Society, is given in alternate years for "meritorious distinction in Science, Literature, Industry or Public Affairs, and who is prominently concerned with the Welfare of the Society". The presentation will be made at the annual meeting of the Society to be held in London on July 9, when Lord Samuel will deliver an address.

The Kitasato Institute for Infectious Diseases, founded at Tokyo, by Baron Kitasato, who discovered the bacillus of bubonic plague and who died in 1931, celebrated the twenty-fifth anniversary of its foundation on November 5 .

According to Dr. C. Louis Leipoldt, medical secretary of the Medical Association of South Africa, in Southern Rhodesia where there are 188 registered medical practitioners; 116 are available for service in the present national emergeney. 